\title{
Bayesian $t$ tests for accepting and rejecting the null hypothesis
}

\author{
Jeffrey N. Rouder, Paul L. Speckman, Dongchu Sun, and Richard D. Morey \\ University of Missouri, Columbia, Missouri
}

AND

GEOFFREY IVERSON

University of California, Irvine, California

\begin{abstract}
Progress in science often comes from discovering invariances in relationships among variables; these invariances often correspond to null hypotheses. As is commonly known, it is not possible to state evidence for the null hypothesis in conventional significance testing. Here we highlight a Bayes factor alternative to the conventional $t$ test that will allow researchers to express preference for either the null hypothesis or the alternative. The Bayes factor has a natural and straightforward interpretation, is based on reasonable assumptions, and has better properties than other methods of inference that have been advocated in the psychological literature. To facilitate use of the Bayes factor, we provide an easy-to-use, Web-based program that performs the necessary calculations.
\end{abstract}

Advances in science often come from identifying invariances - those elements that stay constant when others change. Kepler, for example, described the motion of planets. From an Earth-bound vantage point, planets seem to have strange and variable orbits. Not only do they differ in their speeds and locations, they even appear to backtrack at times (a phenomenon known as retrograde motion). Although planetary orbits appear variable, Kepler identified invariants in planetary motion. For example, all orbits follow ellipses in which the square of the orbital period is proportional to the cube of the orbital radius. These invariances formed the basis for Newton's subsequent theory of mechanics (Hawking, 2002). A similar story holds in genetics, where Mendel's discovery of invariant ratios in phenotypes served as an important precursor for the construction of genetic theory.

Although the search for invariances has often motivated theory in other domains, it has not had as much impact in psychology. Invariances are statements of equality, sameness, or lack of association, whereas in practice, the psychological field has a Popperian orientation, in which demonstrations of effects or associations are valued more than demonstrations of invariances (Meehl, 1978). As a contrast, we offer below a few examples of how scientific inquiry in cognitive psychology has benefited from consideration of invariances:

1. It is often of great practical and theoretical interest to determine whether performance is invariant to readily observable variables. For example, several researchers have assessed whether cognitive skills vary with gender (e.g.,
Shibley Hyde, 2005, 2007). To believe that only effects of genders, rather than invariances across genders, will appear in performance strikes us as an extreme position. A second example comes from the domain of subliminal priming (see, e.g., Dehaene et al., 1998): To prove that subliminal priming occurs, it must be shown that detection or identification of the primes does not vary from chance (see Reingold \& Merikle, 1988; Rouder, Morey, Speckman, \& Pratte, 2007).

2. Conservation laws are instantiations of invariances. An example of a proposed conservation law is the WeberFechner law (Fechner, 1860/1966), which states that the detectability of a briefly flashed stimulus is a function of its intensity divided by the intensity of the background. Accordingly, performance should be invariant when the intensities of the flash and background are multiplied by the same constant. Another example of a proposed conservation law is the choice rule (Clarke, 1957; Luce, 1959; Shepard, 1957), which states that the preference for a choice is a function of the ratio of its utility divided by the summed utility of all available choices. The key invariance here concerns ratios of preferences between any two choices - for example, the preference for Choice A divided by that for Choice B. This ratio should not vary when choices are added or taken away from the set of available options.

3 . Testing invariances is critical for validating parametric descriptions. Consider the example of Stevens (1957), who proposed that sensation follows a power function of intensity. It is reasonable to expect that the exponent of

J. N. Rouder, rouderj@missouri.edu 
the power function will vary across variables of different intensities, such as brightness or loudness. As pointed out by Augustin (2008), however, it is critical that the exponent be constant for a given-intensity variable. Exponents should not, for instance, depend on the specific levels chosen in an experiment. Another example of a parametric description is from Logan $(1988,1992)$, who proposed that response time (RT) decreases with practice as a power function. For this description to be valid, an invariance in the exponent across various experimental elements would be expected (e.g., in alphabet arithmetic, the power should be invariant across addends).

4. Assessing invariances is also critical for showing selective influence, which is a key method of benchmarking formal models. Debner and Jacoby (1994), for instance, tested Jacoby's (1991) process dissociation model by manipulating attention at study in a memory task. This manipulation should affect parameters that index conscious processes but not those that index automatic ones. Similarly, the theory of signal detection (Green \& Swets, 1966) stipulates that base-rate manipulations affect criteria but not sensitivity. Hence, an invariance of sensitivity over this manipulation is to be expected (Egan, 1975; Swets, 1996).

This small, selective set of examples demonstrates the appeal of testing invariances for theory building. Even so, it is worth considering the argument that invariances do not exist, at least not exactly. Cohen (1994), for example, started with the proposition that all variables affect all others to some, possibly small, extent. Fortunately, there is no real contradiction between adhering to Cohen's view that invariances cannot hold exactly and assessing invariances for theory building. The key here is that invariances may not hold exactly for relatively trivial reasons that are outside the domain of study. When they hold only approximately, they often provide a more parsimonious description of data than do the alternatives and can serve as guidance for theory development. Hence, whether one believes that invariances may hold exactly or only approximately, the search for them is intellectually compelling.

Perhaps the biggest hurdle to assessing invariances is methodological. Invariances correspond to the null hypothesis of equality, but conventional significance tests do not allow the analyst to state evidence for a null hypothesis. If an invariance holds, even approximately, then the best-case significance test outcome is a failure to reject, which is interpreted as a state of ignorance. In this article, we recommend Bayes factors (Kass \& Raftery, 1995) as a principled method of inference for assessing both invariances and differences. Bayes factors have been recommended previously in the psychological literature (see, e.g., Edwards, Lindman, \& Savage, 1963; Lee \& Wagenmakers, 2005; Myung \& Pitt, 1997; Wagenmakers, 2007). In this article, we develop Bayes factor tests for paired (one-sample) and grouped (two-sample) $t$ tests. Moreover, we provide a freely available, easy-to-use, Web-based program for this analysis (available at pcl.missouri.edu). We anticipate that the approach presented here will generalize to factorial designs across several variables.

\section{Critiques of Inference by Significance Tests}

Before introducing Bayes factors, we present two related critiques of classic null-hypothesis significance tests: (1) They do not allow researchers to state evidence for the null hypothesis, and, perhaps more importantly, (2) they overstate the evidence against the null hypothesis. To make these critiques concrete, assume that each of $N$ participants performs a task in two different experimental conditions. Let $x_{i 1}$ and $x_{i 2}$ denote the $i$ th participant's mean RT in each of the two conditions, and let $y_{i}$ denote the difference between these mean RTs. A typical test of the effect of conditions is a one-sample $t$ test to assess whether the population mean of $y_{i}$ is different from zero. The model underlying this test is

$$
y_{i} \stackrel{\text { iid }}{\sim} \operatorname{Normal}\left(\mu, \sigma^{2}\right), i=1, \ldots, N .
$$

The null hypothesis, denoted $H_{0}$, corresponds to $\mu=0$. The alternative, denoted $H_{1}$, corresponds to $\mu \neq 0$.

The first critique, that null-hypothesis significance tests do not allow the analyst to state evidence for the null hypothesis, can be seen by considering how $p$ values depend on sample size. Significance tests seem quite reasonable if the null is false. In this case, the $t$ values tend to become larger as sample size is increased; this in turn increases the probability of correctly rejecting the null. In the largesample limit - that is, as the sample size becomes arbitrarily large - the $t$ value grows without bound, and the $p$ value converges to zero. This behavior is desirable, because it implies that the null will always be rejected in the large-sample limit when the null is false. Researchers, therefore, can rest assured that increasing sample size will, on average, result in a gain of evidence against the null when the null is, indeed, false.

The situation is less desirable, however, if the null is true. When the null is true, the $t$ values do not converge to any limit with increasing sample size. For sample sizes greater than 30 or so, the distribution of $t$ values is well approximated by a standard normal distribution. Corresponding $p$ values are also distributed; when the null is true, all $p$ values are equally likely - that is, they are distributed uniformly between 0 and 1 . This distribution holds regardless of sample size. The consequence of this fact is that researchers cannot increase the sample size to gain evidence for the null, because increasing the sample size does not affect the distribution of $p$ values. Of course, this behavior is part of the design of significance tests and reflects Fisher's view that null hypotheses are only to be rejected and never accepted (Meehl, 1978).

One pernicious and little-appreciated consequence of the inability to gain evidence for the null is that significance tests tend to overstate the evidence against it (Edwards et al., 1963; Goodman, 1999; Jeffreys, 1961; Sellke, Bayarri, \& Berger, 2001; Wagenmakers \& Grünwald, 2006). A rejection of the null hypothesis may be an exaggeration of the evidence for an effect. To show this overstatement, we start by considering the behavior of significance tests in the large-sample limit. It is reasonable to expect that in this limit, a method of inference always yields the correct answer. In statistics, this property is 
termed consistency, and inconsistent inferential methods strike us as unsatisfying. If the null is false $(\mu \neq 0)$, significance tests are consistent: The $p$ values converge to zero, and the null is always rejected. If the null is true $(\mu=0)$, however, significance tests are inconsistent: Even in the large-sample limit, the analyst mistakenly rejects the null with probability $\alpha$. This inconsistency is a bias to overstate the evidence against the null. This bias would be of only theoretical interest if it held exclusively in the largesample limit. But in fact, it holds for realistic sample sizes as well, and therefore is of practical concern. To show this bias in realistic sample sizes, we present an argument motivated by that of Sellke et al.

Suppose that each of 100 participants provides 100 RT measurements in each of two conditions. Let us assume that RTs for each individual in each condition have a standard deviation (std) of $300 \mathrm{msec}$, which is reasonable for RTs with means between 500 and $1,000 \mathrm{msec}$. The analyst tabulates participant-by-condition means, denoted $x_{1 i}$ and $x_{2 i}$. These participant-by-condition means have standard deviations of $300 / \sqrt{ } 100=30$. From these means, participant difference scores, $y_{i}=x_{2 i}-x_{1 i}$, are calculated, and the standard deviation ${ }^{1}$ of $y_{i}$ is $\sqrt{ } 2 \times 30 \approx 42$. Let us assume that the difference scores reveal a $\bar{y}=10$-msec RT advantage for one experimental condition over the other. The $t$ statistic is $t=\sqrt{ } N \times \bar{y} / \operatorname{std}(y)=10 \times 10 / 42=2.38$. The associated $p$ value is about .02 . The conventional wisdom is that this is a case in which the null hypothesis is not tenable. We show below that this conclusion is an overstatement.

One way of quantifying the evidence in the data is to compute the likelihood of observing a $t$ value of 2.38 under various hypotheses. The likelihood of $t=2.38$ for the null is the density of a $t$ distribution with 99 degrees of freedom evaluated at 2.38. This likelihood is small, about .025. Differences in RTs tend to vary across tasks, but even so, it is common for reliable differences to be $30 \mathrm{msec}$ or more. The likelihood that $t=2.38$ for the more typical difference of $30 \mathrm{msec}$ is the density of a noncentral $t$ distribution with 99 degrees of freedom and a noncentrality parameter of $(\sqrt{ } N) \mu / \sigma$, which is about 7.1. This likelihood is quite small, about .000006 . The likelihood ratio of the null versus this typical effect is about 3,800:1 in favor of the null. Hence, a likelihood ratio test of the null versus a typical effect strongly favors the null.

Figure 1 shows this likelihood ratio for a range of alternatives. The filled square shows the likelihood ratio for the example above of the null hypothesis versus a typical effect of $30 \mathrm{msec}$. The null has greater likelihood for all effects greater than about $20 \mathrm{msec}$. The fact that there is a large range of reasonable alternatives against which the null is preferable is not captured by the $p$ value. In view of this fact, rejection of null hypotheses by consideration of $p$ values under the null strikes us, as well as several other commentators, as problematic.

There are two related lessons from the demonstration above. The first is that the choice of alternative matters. The evidence in the example above provides different support for the null depending on the alternative. It favors the

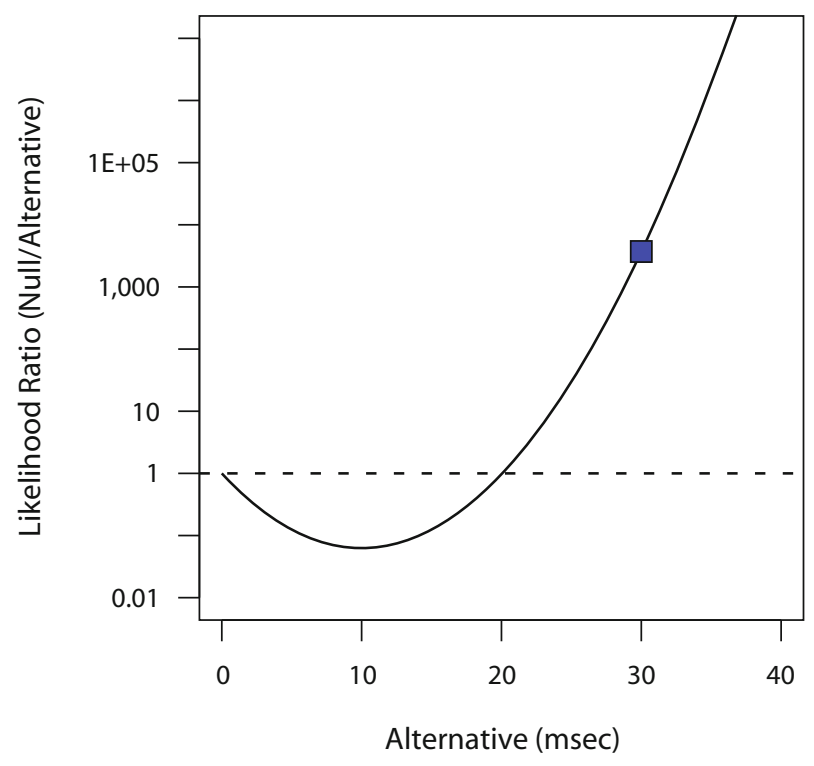

Figure 1. Likelihood ratio (null/alternative) as a function of the alternative for a hypothetical data set $(t=2.38,100$ participants observing 100 trials in each of two conditions). Even though the null is rejected by a standard significance test, there is not strong evidence against it as compared with the specific alternative.

null if the alternative is typical $(\mu=30)$ but favors the alternative if the alternative is a small effect $(\mu=10)$. It is an undeniable fact that inference depends on how much weight the researcher places on various alternatives. Subsequently, we will discuss strategies for weighting alternatives. The second lesson is that significance tests overstate the evidence against the null in finite samples because the null may be more plausible than other reasonable alternatives. As a rule of thumb, inference based on evaluating a null without comparison to alternatives tends to overstate the evidence against the null. Some examples of this tendency include:

Confidence intervals (CIs). Cumming and Finch (2001), Masson and Loftus (2003), and Kline (2004), among many others, have recommended using CIs for inference. We recommend CIs for reporting data, but not for inference (Rouder \& Morey, 2005). The problem with confidence intervals for inference is that they, like significance tests, have a fixed probability of including the null when the null is true, regardless of sample size. This property implies that evidence cannot be gained for the null, and, consequently, there is a tendency to overstate the evidence against it. For the one-sample case, the null is rejected if the $\mathrm{CI}$ around the mean does not include zero. This test is, of course, equivalent to significance testing and suffers analogously to the example above.

Probability of replication $\left(p_{\text {rep }}\right)$. Killeen $(2005,2006)$ recommends computing the predictive posterior probability that a different sample from the same experiment would yield an effect with the same sign as the original. Inference is performed by rejecting the null if this probability is sufficiently large. Although this approach may appear attractive, it is not designed to assess the replica- 
bility of invariances. As discussed by Wagenmakers and Grünwald (2006), $p_{\text {rep }}$ is logically similar to $p$ values in that the distribution does not change with sample size when the null holds. As such, it is open to the critiques of $p$ values above.

Neyman-Pearson (NP) hypothesis testing with fixed $\alpha$. In NP testing, the researcher may specify an alternative to the null and then choose a decision criterion on the basis of consideration of Type I and Type II error rates. Typically, however, psychologists fix the decision criterion with reference to the null alone - that is, with fixed $\alpha$, say $\alpha=$ .05 . This choice is not necessitated by NP testing per se; instead, it is a matter of convention in practice. With this choice, NP is similar to Fisher significance testing based on $p$ values, at least for many common tests (see Lehmann, 1993, for details). Consequently, it is similarly biased toward overstating the evidence against the null. This point is made elegantly by Raftery (1995). NP testing can be made consistent by allowing Type I error rates to decrease toward zero as the sample size increases. How this rate should decrease with sample size, however, is neither obvious nor stipulated by the statistical theory underlying NP testing.

The Akaike information criterion (AIC). The AIC (Akaike, 1974) is a method of model selection (rather than testing) that is occasionally used in psychology (examples include Ashby \& Maddox, 1992; Rouder \& Ratcliff, 2004). One advantage of the AIC is that it seemingly may be used to state evidence for the null hypothesis. To use the AIC, each model is given a score:

$$
\mathrm{AIC}=-2 \log L+2 k,
$$

where $L$ is the maximum likelihood under the model and $k$ is the number of required parameters. The model with the lowest AIC is preferred. AIC, however, has a bias to overstate the evidence against the null. This bias is easily seen in the large-sample limit for the one-sample case. If the AIC is consistent, the Type I error rate should decrease to zero in the large-sample limit. According to AIC, the alternative is preferred when $-2 \log \left(L_{1}-L_{0}\right)>2$, and the Type I error rate is the probability of this event when the null is true. Under the null, the quantity $-2 \log \left(L_{1}-L_{0}\right)$ is asymptotically distributed as a chi-square with one degree of freedom (see Bishop, Fienberg, \& Holland, 1975). The Type I error rate in the limit is therefore the probability that this chi-square distribution is greater than 2.0, which is about .157 rather than 0 .

In summary, conventional significance tests do not allow the researcher to state evidence for the null. Hence, they are not appropriate for competitively testing the null against the alternative. As mentioned previously, invariances may play a substantial role in theory building. Hence, methods for testing them are needed.

\section{Bayes Factors}

We advocate inference by Bayes factors (Jeffreys, 1961; Kass \& Raftery, 1995). This method is logically sound and yields a straightforward and natural interpretation of the evidence afforded by data. In Bayesian statistics, it is possible to compute the probability of a hypothesis conditionally on observed data. Quantities that are conditional on observed data are termed posterior; an appropriate statistic for comparing hypotheses is the posterior odds:

$$
\Omega=\frac{\operatorname{Pr}\left(H_{0} \mid \text { data }\right)}{\operatorname{Pr}\left(H_{1} \mid \text { data }\right)},
$$

where $H_{0}$ and $H_{1}$ denote the null and alternative hypotheses, respectively. Odds are directly interpretable. For instance, if $\Omega=19$, the null is 19 times more probable than the alternative, given the data. As Laplace first noted almost 200 years ago, computing posterior odds on hypotheses is natural for scientific communication (as cited in Gillispie, Fox, \& Grattan-Guinness, 1997, p. 16). Jeffreys recommends that odds greater than 3 be considered "some evidence," odds greater than 10 be considered "strong evidence," and odds greater than 30 be considered "very strong evidence" for one hypothesis over another.

The posterior odds are given by

$$
\Omega=\frac{\operatorname{Pr}\left(H_{0} \mid \text { data }\right)}{\operatorname{Pr}\left(H_{1} \mid \text { data }\right)}=\frac{f\left(\text { data } \mid H_{0}\right)}{f\left(\text { data } \mid H_{1}\right)} \times \frac{\operatorname{Pr}\left(H_{0}\right)}{\operatorname{Pr}\left(H_{1}\right)} .
$$

The term $\operatorname{Pr}\left(H_{0}\right) / \operatorname{Pr}\left(H_{1}\right)$ is the prior odds. In practice, it is often natural to set the prior odds to 1.0 , a position that favors neither the null nor the alternative. The terms $f\left(\right.$ data $\left.\mid H_{0}\right)$ and $f\left(\right.$ data $\left.\mid H_{1}\right)$ are called the marginal likelihoods and are denoted more succinctly as $M_{0}$ and $M_{1}$, respectively. The posterior odds is, therefore,

$$
\Omega=\frac{M_{0}}{M_{1}} \times \frac{\operatorname{Pr}\left(H_{0}\right)}{\operatorname{Pr}\left(H_{1}\right)} .
$$

All of the evidence from the data is expressed in the ratio of marginal likelihoods. This ratio is termed the Bayes factor (Jeffreys, 1961; Kass \& Raftery, 1995) and is denoted by $B_{01}$ :

$$
B_{01}=\frac{M_{0}}{M_{1}}
$$

Consequently,

$$
\Omega=B_{01} \times \frac{\operatorname{Pr}\left(H_{0}\right)}{\operatorname{Pr}\left(H_{1}\right)} .
$$
by

Marginal likelihoods for a given hypothesis $H$ are given

$$
M_{H}=\int_{\boldsymbol{\theta} \in \Theta_{H}} f_{H}(\boldsymbol{\theta} ; \mathbf{y}) p_{H}(\boldsymbol{\theta}) d \boldsymbol{\theta},
$$

where $\Theta_{H}$ denotes the parameter space under hypothesis $H, f_{H}$ denotes the probability density function of the data under hypothesis $H, \mathbf{y}$ denotes the data, and $p_{H}$ denotes the prior distribution on the parameters. This equation is most profitably viewed as a continuous average of likelihoods in which priors $p_{H}$ serve as the weights. If a prior places weight on parameter values that are very unlikely to have produced the data, the associated low likelihood values will drag down the average. Hence, in order for the marginal likelihood of a model to be competitive, the prior should not attribute undue mass to unreasonable parameter values. As will be discussed in the next section, this 
fact is important in understanding why analysts must commit to reasonable alternatives for principled inference.

For the one-sample application, the null model has a single parameter, $\sigma^{2}$, and the alternative model has two parameters, $\sigma^{2}$ and $\mu$. The marginal likelihoods are

$$
M_{0}=\int_{0}^{\infty} f_{0}\left(\mathbf{y} \mid \sigma^{2}\right) p_{0}\left(\sigma^{2}\right) d \sigma^{2}
$$

and

$$
M_{1}=\int_{-\infty}^{\infty} \int_{0}^{\infty} f_{1}\left(\mathbf{y} \mid \mu, \sigma^{2}\right) p_{1}\left(\mu, \sigma^{2}\right) d \sigma^{2} d \mu
$$

For the one-sample case, priors are needed on $\sigma^{2}$ to describe the null hypothesis. Likewise, priors are needed on $\mu$ and $\sigma^{2}$ to describe the alternative. There are two different schools of thought on how to choose priors in Bayesian analysis. According to the first, subjective Bayes, school, priors should reflect the analyst's a priori beliefs about parameters. These beliefs are informed by the theoretical and experimental context. According to the second, objective Bayes, school, priors should reflect as few assumptions as possible. In this article, we take the objective approach; that is, we seek priors that reflect a minimum degree of information.

\section{The Role of Priors}

In this section, we discuss how the choice of priors affects the resulting Bayes factor. This discussion directly motivates our recommendations and serves as a basis for interpreting and understanding Bayes factors.

It is easiest to build intuition about the role of the priors in a simple case. Assume that $\sigma^{2}$ is known and the alternative is a point much like the null. The null is given by $\mu=0$; the alternative is given by $\mu=\mu_{1}$, where $\mu_{1}$ is specified. Figure 2A shows the effect of the choice of the alternative, $\mu_{1}$ (measured in units of $\sigma$ ), on the Bayes factor for a few values of $\bar{y}$ (also measured in units of $\sigma$ ) when $N=100 .^{2}$ This simple example illustrates that as the alternative is placed farther from the observed data, the Bayes factor increasingly favors the null. Moreover, when the alternative is unrealistically far from the data, the Bayes factor provides unbounded support for the null hypothesis over this alternative. This insight that unrealistic alternatives yield support for the null will be utilized in specifying appropriate alternatives.

In the example above, we assumed that the alternative was at a single point. This assumption, however, is too restrictive to be practical. Instead, it is more realistic to consider an alternative that is a distribution across a range of outcomes. For example, we may place a normal prior on $\mu$ :

$$
\mu \sim \operatorname{Normal}\left(0, \sigma_{\mu}^{2}\right) \text {. }
$$

The normal is centered around zero to indicate no prior commitment about the direction of effects. To use this normal prior, the analyst must set $\sigma_{\mu}^{2}$ a priori. The critical question is how the choice of $\sigma_{\mu}^{2}$ affects the Bayes factor. ${ }^{3}$ One might set $\sigma_{\mu}^{2}=\infty$, which specifies no prior information about $\mu$. In fact, this setting is used as a noninformative prior in Bayesian estimation of $\mu$ (see, e.g., Rouder \& Lu, 2005).

Figure 2B shows the effect of the choice of $\sigma_{\mu}^{2}$ (measured in units of $\sigma$ ) on the resulting Bayes factor for a few values of $\bar{y}$ (also measured in units of $\sigma$ ) when $N=100$. As $\sigma_{\mu}^{2}$ becomes large, the value of $B_{01}$ increases, too. To understand why this behavior occurs, it is helpful to recall that the marginal likelihood of a composite hypothesis is the weighted average of the likelihood over all constituent point hypotheses, where the prior serves as the weight. As
A

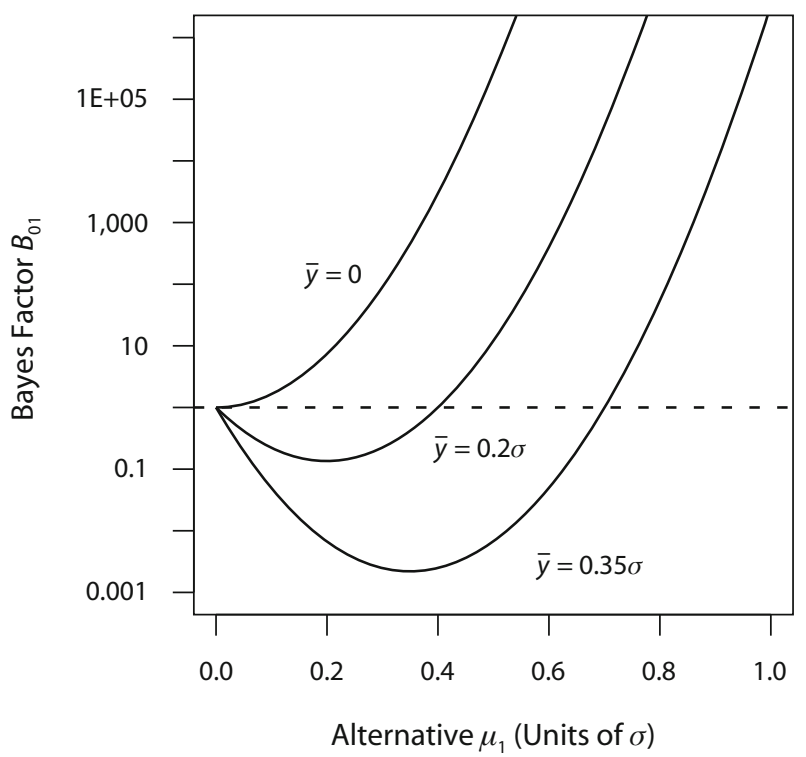

B

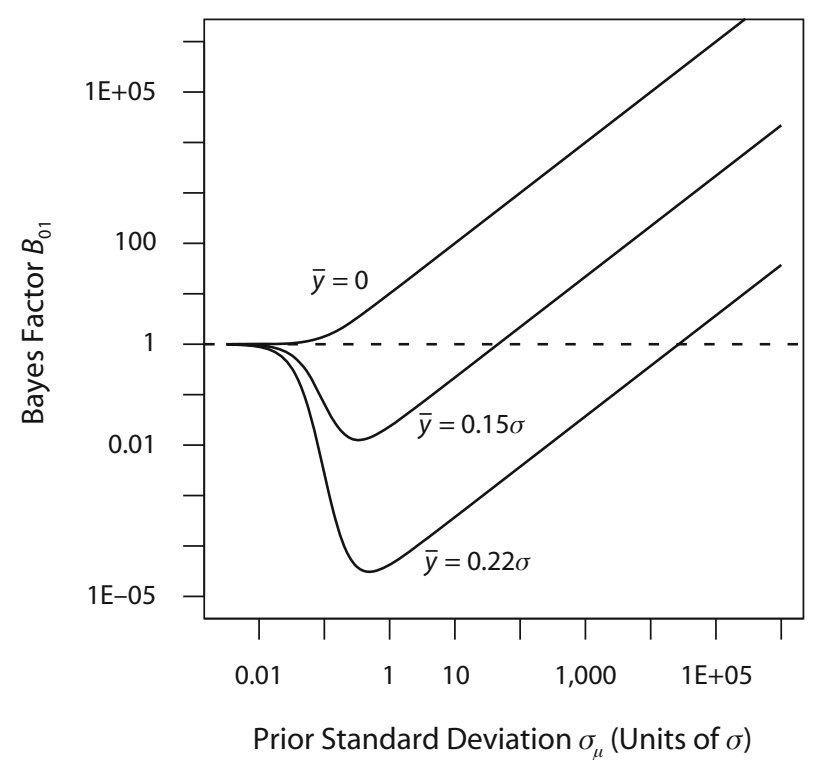

Figure 2. (A) Bayes factors as a function of the alternative. (B) Bayes factors as a function of prior standard deviation $\sigma_{\mu}$ for a normally distributed alternative. Lines depict different sample means (measured in units of standard deviation $\sigma$ ). 
A

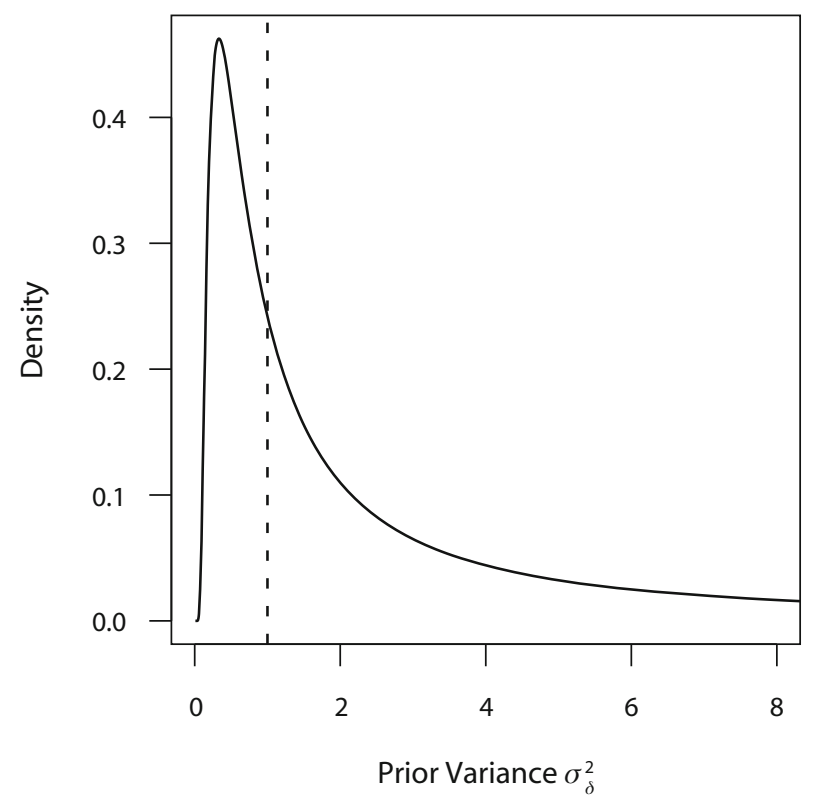

B

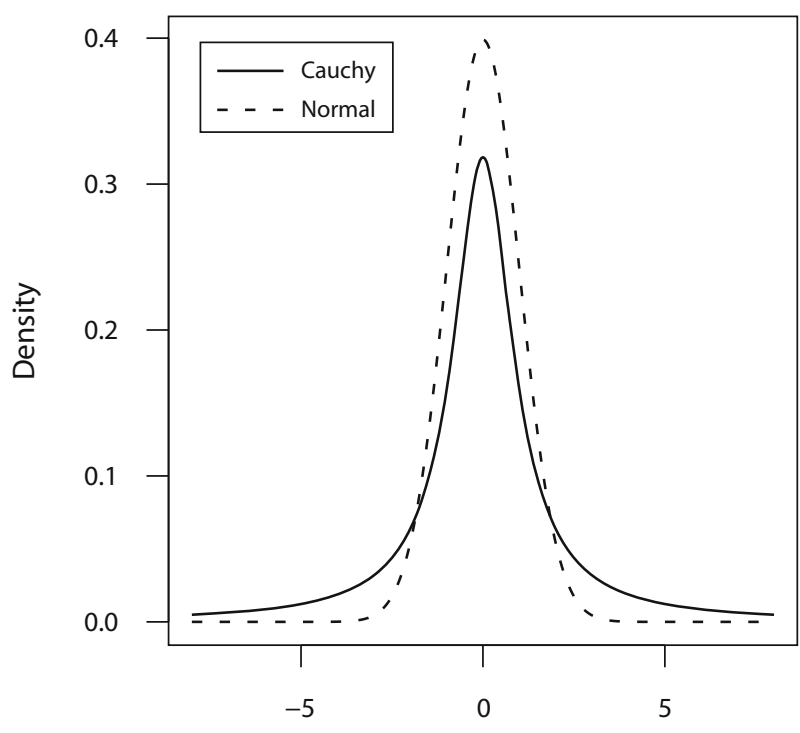

Effect Size $\delta$

Figure 3. (A) Density of the inverse-chi-square prior distribution on $\sigma_{\delta^{*}}^{2}$ (B) Densities of the Cauchy (solid line) and normal (dashed line) prior distributions on effect size $\delta$.

$\sigma_{\mu}^{2}$ is increased, there is greater relative weight on larger values of $\mu$. Unreasonably large values of $\mu$ under the alternatives provide increased support for the null (as shown in Figure 2A). When these unreasonably large values of $\mu$ have increasing weight, the average favors the null to a greater extent. Hence, specifications of alternatives that weight unreasonably large effects heavily will yield Bayes factors that too heavily support the null. Moreover, the setting of $\sigma_{\mu}^{2}=\infty$ implies that the Bayes factor provides unbounded support for the null, a fact known as the JeffreysLindley paradox (Lindley, 1957). Therefore, arbitrarily diffuse priors are not appropriate for hypothesis testing.

To use the normal prior for the alternative, the researcher must specify reasonable values for the variance $\sigma_{\mu}^{2}$. One approach is to customize this choice for the paradigm at hand. For example, the choice $\sigma_{\mu}=20 \mathrm{msec}$ may be reasonable for exploring small effects such as those in priming experiments. The value of $\sigma_{\mu}$ should be greater with more-variable data, such as those from complicated tasks or from clinical populations.

A well-known and attractive alternative to placing priors on the mean $\mu$ is to place them on effect size, where effect size is denoted by $\delta$ and given as $\delta=\mu / \sigma$. The null hypothesis is $\delta=0$. Alternatives may be specified as a normal prior on effect size:

$$
\delta \sim \operatorname{Normal}\left(0, \sigma_{\delta}^{2}\right),
$$

where $\sigma_{\delta}^{2}$ is specified a priori (Gönen, Johnson, $\mathrm{Lu}, \&$ Westfall, 2005). Reparameterizing the model in terms of effect size $\delta$ rather than mean $\mu$ does not change the basic nature of the role of the prior. If $\sigma_{\delta}^{2}$ is set unrealistically high, the Bayes factor will favor the null too heav- ily. The advantage of this effect-size parameterization is that researchers have an intrinsic scale about the ranges of effect sizes that applies broadly across different tasks and populations. For instance, effect sizes of 1.0 are large; those of .02 are very small. Importantly, we can use this knowledge to avoid placing too much weight on unreasonable effect-size values. For instance, we can all agree that priors that place substantial mass on effect sizes over 6 are unrealistic; if a phenomenon yielded such large effect sizes, it would be so obvious as to make experiments hardly necessary.

One reasonable setting is $\sigma_{\delta}^{2}=1$. The corresponding prior on effect size, a standard normal, is shown in Figure 3B (dashed line) and is known as the unit-information prior. The setting is reasonable because the distribution of effect sizes under the alternative does not include much mass on highly implausible effect sizes such as 6 . One advantage of this setting is that small effects are assumed to occur with greater frequency than large ones, which is in accordance with what experimentalists tend to find. With this setting, it can be shown that the alternative has only a small amount of information - in fact, the amount in any single observation (see Kass \& Wasserman, 1995). In fact, the unit-information prior underlies the Bayesian information criterion (BIC; Raftery, 1995; Schwarz, 1978).

With the normal prior, the analyst commits to a single, specific value for $\sigma_{\delta}^{2}$, such as $\sigma_{\delta}^{2}=1$. There is, however, an even less informative formulation: Instead of setting $\sigma_{\delta}^{2}$ to a single value, it can be allowed to range over a distribution of values. Zellner and Siow (1980) recommend the following prior distribution for $\sigma_{\delta}^{2}$ itself:

$$
\sigma_{\delta}^{2} \sim \text { inverse chi-square(1). }
$$


The inverse chi-square family provides useful priors in Bayesian statistics (see, e.g., Gelman, Carlin, Stern, \& Rubin, 2004). The density function of the inverse chisquare with one degree of freedom is shown in Figure 3A. Mass falls off for very small and very large values of $\sigma_{\delta}^{2}$; that is, $\sigma_{\delta}$ is constrained to be somewhat near 1.0. This specification is less informative than the previously discussed unit-information prior, which requires $\sigma_{\delta}^{2}=1$.

Even though we explicitly place a prior on $\sigma_{\delta}^{2}$, there is a corresponding prior on effect size obtained by integrating out $\sigma_{\delta}^{2}$. Liang, Paulo, Molina, Clyde, and Berger (2008) noted that placing a normal on effect size with a variance that is distributed as an inverse chi-square is equivalent to placing the following prior on effect size:

$$
\delta \sim \text { Cauchy. }
$$

The Cauchy distribution is a $t$ distribution with a single degree of freedom. It has tails so heavy that neither its mean nor its variance exist. A comparison of the Cauchy prior to the unit-information prior is shown in Figure 3B. As can be seen, the Cauchy allows for more mass on large effects than the standard normal. Consequently, Bayes factors with the Cauchy prior favor the null a bit more than those with the unit-information prior. It is important to note that the Cauchy was not assumed directly; it results from the assumption of a normal distribution on $\delta$ with variance $\sigma_{\delta}^{2}$ distributed as an inverse chi-square. Jeffreys (1961) was the first to consider the Cauchy specification of the alternative for Bayes factor calculations.

In the preceding analyses, we assumed that $\sigma^{2}$, the variability in the data, is known. Fortunately, it is relatively easy to relax this assumption. The intuition from the preceding discussion is that priors on parameters cannot be too variable, because they then include a number of unreasonable values that, when included in the average, lower the marginal likelihood. This intuition is critical for comparisons of models when a parameter enters into only one of the models, as is the case for parameter $\delta$. It is much less critical when the parameter in question enters into both models, as is the case for parameter $\sigma^{2}$. In this case, having mass on unrealistic values lowers the marginal likelihood of both models, and this effect cancels in the Bayes factor ratio. For the one- and two-sample cases, a very broad noninformative prior on $\sigma^{2}$ is possible. We make a standard choice: $p\left(\sigma^{2}\right)=1 / \sigma^{2}$. This prior is known as the Jeffreys prior on variance (Jeffreys, 1961). The justification for this choice, though beyond the scope of this article, is provided in all Bayesian textbooks.

With this choice for $\sigma^{2}$, the specification of priors is complete. We refer to the combination of the Cauchy on effect size and the Jeffreys prior on variance as the JZS prior, in order to acknowledge the contributions of Jeffreys and of Zellner and Siow. The JZS prior serves as the objective prior for the one- and two-sample cases.

\section{A Bayes-Factor One-Sample $t$ Test}

In the previous section, we outlined the JZS and unitinformation priors, with the JZS prior being noninformative for the one-sample case. In this section, we present and discuss the Bayes factors for the JZS prior. The first step in derivation is to compute marginal likelihoods $M_{0}$ and $M_{1}$ (by averaging the likelihoods with weights given by the priors). These marginal likelihoods are then divided to yield the Bayes factor. At the end of this process, Equation 1 (below) results for the Bayes factor, where $t$ is the conventional $t$ statistic (see, e.g., Hays, 1994), $N$ is the number of observations, and $v=N-1$ is the degrees of freedom. We refer to Equation 1 as the JZS Bayes factor for the one-sample problem. We recommend this JZS Bayes factor as a default for conducting Bayesian $t$ tests.

To our knowledge, Equation 1 is novel. The derivation is straightforward and tedious and not particularly informative. Gönen et al. (2005) provided the analogous equation for the unit-information Bayes factor. Liang et al. (2008) provided the corresponding JZS Bayes factors for testing slopes in a regression model.

Although Equation 1 may look daunting, it is simple to use. Researchers need only provide the sample size $N$ and the observed $t$ value. There is no need to input raw data. The integration is over a single dimension and is computationally straightforward. We provide a freely available Web-based program that computes the JZS Bayes factor for input values of $t$ and $N$ (pcl.missouri.edu). It also computes the unit-information Bayes factor-that is, the Bayes factor when the unit-information prior is assumed.

Table 1 provides critical $t$ values needed for JZS Bayes factor values of $1 / 10,1 / 3,3$, and 10 as a function of sample size. This table is analogous in form to conventional $t$ value tables for given $p$ value criteria. For instance, suppose a researcher observes a $t$ value of 3.3 for 100 observations. This $t$ value favors the alternative and corresponds to a JZS Bayes factor less than $1 / 10$ because it exceeds the critical value of 3.2 reported in the table. Likewise, suppose a researcher observes a $t$ value of 0.5 . The corresponding JZS Bayes factor is greater than 10 because the $t$ value is smaller than 0.69 , the corresponding critical value in Table 1. Because the Bayes factor is directly interpretable as an odds ratio, it may be reported without reference to cutoffs such as 3 or $1 / 10$. Readers may decide the meaning of odds ratios for themselves.

Figure 4 shows the critical $t$ value needed for JZS Bayes factors of $1 / 10$, a substantial amount of evidence in favor of the alternative (solid line). For large sample sizes, in-

$$
B_{01}=\frac{\left(1+\frac{t^{2}}{v}\right)^{-(v+1) / 2}}{\int_{0}^{\infty}(1+N g)^{-1 / 2}\left(1+\frac{t^{2}}{(1+N g) v}\right)^{-(v+1) / 2}(2 \pi)^{-1 / 2} g^{-3 / 2} e^{-1 /(2 g)} d g},
$$


Table 1

Critical $t$ Values

\begin{tabular}{|c|c|c|c|c|}
\hline \multirow[b]{3}{*}{$N$} & \multicolumn{4}{|c|}{ JZS Bayes Factor Value } \\
\hline & \multicolumn{2}{|c|}{ Favors Null } & \multicolumn{2}{|c|}{$\begin{array}{c}\text { Favors } \\
\text { Alternative }\end{array}$} \\
\hline & 10 & 3 & $1 / 3$ & $1 / 10$ \\
\hline 5 & - & 0.40 & 3.15 & 4.97 \\
\hline 10 & - & 0.89 & 2.73 & 3.60 \\
\hline 20 & - & 1.20 & 2.64 & 3.26 \\
\hline 50 & - & 1.51 & 2.68 & 3.17 \\
\hline 100 & 0.69 & 1.72 & 2.76 & 3.20 \\
\hline 200 & 1.08 & 1.90 & 2.86 & 3.27 \\
\hline 500 & 1.44 & 2.12 & 2.99 & 3.38 \\
\hline
\end{tabular}

creasingly larger $t$ values are needed to maintain the same odds. This behavior ensures that the JZS Bayes factor does not favor the alternative when there are small effects in large samples. The dashed-and-dotted line shows the needed $t$ value for $p=.05$. This $t$ value does not increase with sample size. Also shown is the curve for the unitinformation Bayes factor (longer dashed line), which is derived from the unit-information prior $\left(\sigma_{\delta}^{2}=1\right)$ with a noninformative Jeffreys prior on $\sigma^{2}$ - that is, $p\left(\sigma^{2}\right)=1 / \sigma^{2}$. As can be seen, the unit-information Bayes factor behaves similarly to the JZS Bayes factor. The shorter dashed line is for the BIC, which is discussed subsequently.

Figure 4 reveals that inference with a criterial $p$ value of .05 admits much lower $t$ values as evidence against the null hypothesis than do Bayesian methods with criterial odds of 10:1. This difference has implications in practice. We highlight a few recent examples from the literature in which researchers have rejected the null even though the posterior odds do not indicate that such a rejection is warranted. Grider and Malmberg (2008) assessed whether participants remembered emotional words better than neutral ones in a recognition memory task. In their Experiment 3 , they used a forced choice paradigm in which the targets and lures at test had the same emotional valence. The advantage of this design is that any difference in accuracy could not be due to a response bias for a particular emotional-valence level. Grider and Malmberg claimed that emotional words were remembered better than neutral ones on the basis of two paired $t$ tests on accuracy: one between neutral and positive words [.76 vs. $.80 ; t(79)=$ $2.24]$ and one between neutral and negative words [.76 vs. $.79 ; t(79)=2.03]$. The JZS Bayes factors for these $t$ values and the sample size of $N=80$ may be obtained from the Web-based program. The resulting values are $B_{01}=$ 1.02 and $B_{01}=1.56$ for the two tests, which can only be considered as ambiguous evidence. The latter contrast is especially interesting because the evidence favors the null slightly (odds of 3:2), even though the null is rejected by a significance test with $p<.05$.

Another example comes from Plant and Peruche (2005), who assessed whether a sensitivity training program reduced the likelihood that law enforcement officers mistakenly shot civilians in a computer simulation. They assessed how 48 participating officers performed before and after training. On the basis of a significant onesample $F$ test $[F(1,47)=5.70]$, they concluded that the training was effective. The reported $F$ value corresponds to a $t$ value of 2.39. The JZS Bayes factor for this contrast is 0.63 , which is about 1.6 to 1 in favor of the alternative. These odds, however, do not constitute much evidence for the effectiveness of the training program.

\section{Subjectivity in Priors}

The JZS prior is designed to minimize assumptions about the range of effect size, and in this sense it is an objective prior. In many cases, researchers have knowledge of the domain that may improve inference. This knowledge may be incorporated by changing the form of the prior. On a rather mundane level, a researcher may believe that psychologists tend not to run experiments to search for effects so large that they may be confirmed with 10 or fewer observations. This belief implies that effect sizes much greater than 2.0 in magnitude are improbable, because larger effect sizes would be evident with only a handful of observations. This belief, which strikes us as reasonable in many contexts, might lead some analysts to choose the unit-information Bayes factor over the JZS Bayes factor, since the tails of the normal prior on effect size fall more quickly than those of the Cauchy (see Figure 3B). We recommend that researchers incorporate information when they believe it to be appropriate. If they have no such information or wish not to commit to any, the JZS prior can serve as the noninformative default. The Web-based program also calculates unit-information Bayes factors.

Researchers may also incorporate expectations and goals for specific experimental contexts by tuning the scale of the prior on effect size. The JZS prior on effect

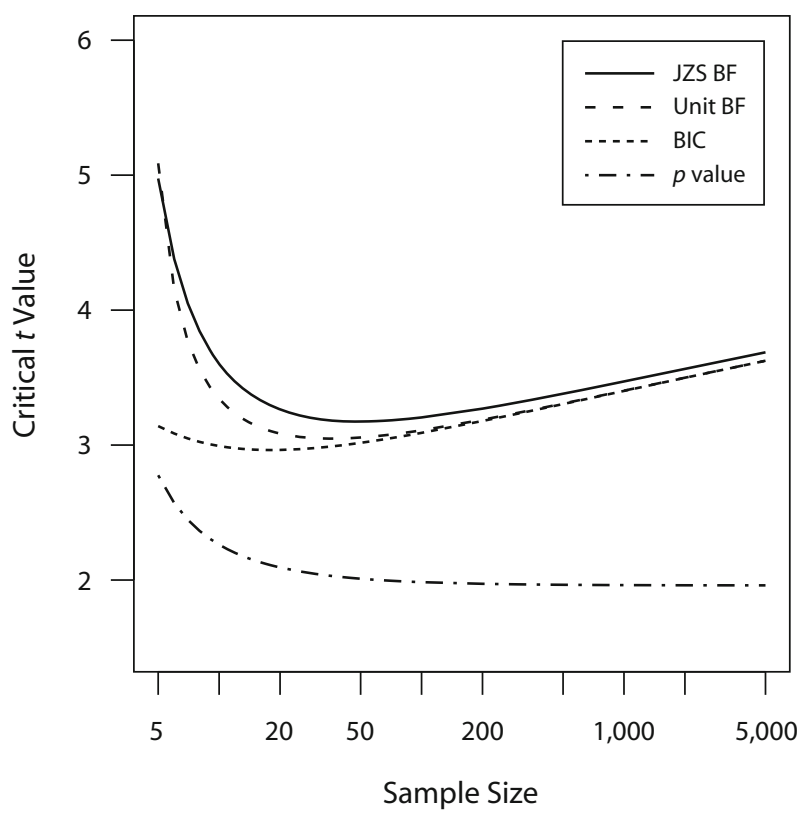

Figure 4. Critical $t$ values needed for posterior odds of 10:1 favoring the alternative for the JZS Bayes factor (solid line), the unit-information Bayes factor (longer dashed line), and the BIC (shorter dashed line), as well as critical $t$ values needed for $p<.05$ (dashed-and-dotted line). 
size can be generalized to $\delta \sim r \times$ Cauchy, where $r$ is a scale factor. ${ }^{4}$ The unit-information prior can be scaled, too: $\delta \sim \operatorname{Normal}\left(0, r^{2}\right)$. In this case, however, the term unit-information prior may be misleading, and we prefer the term scaled-information prior. ${ }^{5}$ For both JZS and scaled-information priors, as $r$ is increased, the Bayes factor provides increased support for the null. In Equation 1, the scale $r$ is implicitly set to 1.0 , which serves as a natural benchmark. Smaller values of $r$, say 0.5 , may be appropriate when small effect sizes are expected a priori; larger values of $r$ are appropriate when large effect sizes are expected. The choice of $r$ may be affected by theoretical considerations, as well: Smaller values are appropriate when small differences are of theoretical importance, whereas larger values are appropriate when small differences most likely reflect nuisances and are of little theoretical importance. In all cases, the value of $r$ should be chosen prior to analysis and without influence from the data. In summary, $r=1.0$ is recommended, though a priori adjustments may be warranted in certain contexts. The aforementioned Web-based program allows researchers to specify $r$, with $r=1.0$ serving as a default.

It may appear that Bayes factors are too dependent on the prior to be of much use. Perhaps researchers can engineer any result they wish by surreptitiously choosing a self-serving prior. This appearance is deceiving. Bayes factors are not particularly sensitive to reasonable variation in priors, at least not with moderate sample sizes. In practice, dramatic changes in the priors often have marginal effects on the results. The previous example of the results from Grider and Malmberg (2008) is useful for making this point. The $t$ value of 2.03 actually corresponded to slight evidence for the null $\left(B_{01}=1.56\right)$, even though the null was rejected at the $p<.05$ level. A reasonable conclusion is that there was not enough evidence in the experiment to express preference for either the null or the alternative hypothesis. The unit-information Bayes factor in this case is 1.21 , which leads to the same conclusion. Suppose we commit a priori to an alternative that is characterized by very small effect sizes for Grider and Malmberg's experiments. Setting $r=0.1$, which seems too low, results in a JZS Bayes factor of 0.59 . Although this value now slightly favors the alternative, it does not support a preference for it. For Grider and Malmberg's data, any reasonable prior leads to the same conclusion, that the evidence does not support a preference. In general, researchers may differ in their choice of priors. If these differences are reasonable, they will have only modest effects on the resulting conclusions.

\section{Bayes Factors With Small Effects}

Previously, we considered the argument that invariances are only true approximately and never exactly (Cohen, 1994). In this section, we explore the behavior of Bayes factors when an invariance holds approximately rather than exactly. As discussed previously, the view that the null can never hold exactly does not negate its usefulness as an idealization. Our main question is whether the Bayes factor provides an appropriate decision about whether the null or the alternative provides a better description of the data for very small true effect sizes.
Figure 5 shows how the Bayes factor depends on sample size for the small true effect size of $\delta=.02$. For small to moderate sample sizes, the Bayes factor supports the null. As the sample size becomes exceedingly large, however, the small deviations from the null are consequential, and the Bayes factor yields less support for the null. In the largesample limit, the Bayes factor favors the alternative, since the null is not exactly true. This behavior strikes us as ideal. With smaller sample sizes that are insufficient to differentiate between approximate and exact invariances, the Bayes factors allows researchers to gain evidence for the null. This evidence may be interpreted as support for at least an approximate invariance. In very large samples, however, the Bayes factor allows for the discovery of small perturbations that negate the existence of an exact invariance. In sum, the Bayes factor favors the more parsimonious nullmodel description with small observed effect sizes unless the sample size is so large that even these small effects are not compatible with the null relative to the alternative. ${ }^{6}$

\section{Extension to Two-Sample Designs}

The Bayes factor in Equation 1 is applicable for onesample designs and is analogous to a paired $t$ test. Onesample designs are common in experimental psychology because variables are often manipulated in a withinsubjects manner. Between-subjects designs are also common and are often used to compare participant variables such as age or gender. For cases with two independent

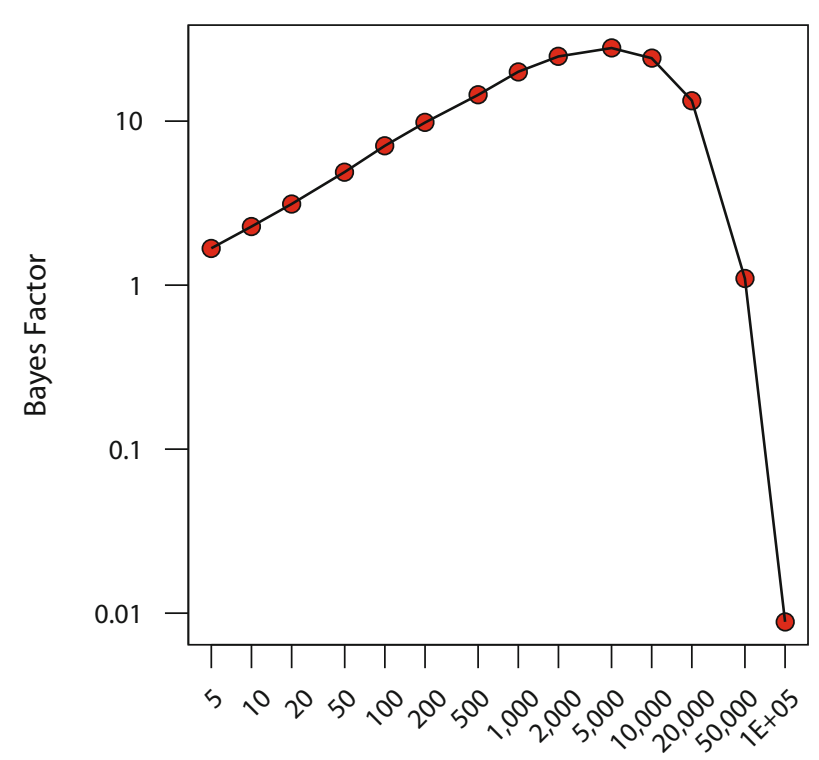

Sample Size

Figure 5. Bayes factors for a small true effect size (.02). Shown is the expected unit-information Bayes factor as a function of sample size. These expected values are obtained by a Monte Carlo simulation in which data are repeatedly sampled from a normal distribution with effect size $\delta=.02$. As shown, the Bayes factor allows researchers to gain evidence for the approximate invariance with sample sizes below 5,000 and to gain evidence for small effects with sample sizes over 50,000 . The JZS Bayes factor acts similarly, although the evaluation of the integral is unstable for sample sizes $>5,000$. 
groups, the two-sample (groups) $t$ test is appropriate. Below is the development of the JZS Bayes factor for the two-sample case.

Let $x_{i}$ and $y_{i}$ denote the $i$ th observations in the first and second groups, respectively. These observations are conventionally modeled as

$$
\begin{aligned}
& x_{i} \stackrel{\text { iid }}{\sim} \operatorname{Normal}\left(\mu-\frac{\alpha}{2}, \sigma^{2}\right), \quad i=1, \ldots, N_{x}, \\
& y_{i} \stackrel{\text { iid }}{\sim} \operatorname{Normal}\left(\mu+\frac{\alpha}{2}, \sigma^{2}\right), \quad i=1, \ldots, N_{y},
\end{aligned}
$$

where $\mu$ and $\alpha$ denote the grand mean and total effect, respectively, and $N_{x}$ and $N_{y}$ denote the sample size for the first and second groups, respectively. The null hypothesis corresponds to $\alpha=0$. As before, it is convenient to consider an effect size, $\delta=\alpha / \sigma$. In this parameterization, the null hypothesis corresponds to $\delta=0$; the JZS prior for $\delta$ under the alternative is given by

$$
\delta \sim \text { Cauchy. }
$$

Priors are needed for $\mu$ and $\sigma^{2}$. Fortunately, these parameters are common to both models, and the resulting Bayes factor is relatively robust to the choice. The Jeffreys noninformative prior on $\sigma^{2}, p\left(\sigma^{2}\right)=1 / \sigma^{2}$, is appropriate. A noninformative prior may also be placed on $\mu$. In this prior, all values of $\mu$ are equally likely; this prior is denoted $p(\mu)=1$.

Equation 1 may be adapted to compute the two-sample JZS Bayes factor with the following three substitutions: (1) The value of $t$ is the observed two-sample (grouped) $t$ value; (2) the effective sample size is $N=N_{x} N_{y} /\left(N_{x}+\right.$ $N_{y}$ ); and (3) the degrees of freedom are $v=N_{x}+N_{y}-2$. Our Web-based program also computes JZS and unit Bayes factors for this two-sample case; the user need only input both sample sizes and the group $t$ value.

\section{Bayesian Information Criterion}

The BIC (Schwarz, 1978) is a Bayesian model selection technique that has been recommended in psychology (see, e.g., Wagenmakers, 2007). In the BIC, each model is given a score:

$$
\mathrm{BIC}=-2 \log L+k \log N,
$$

where $L$ is the maximum likelihood of the model, $N$ is the sample size, and $k$ is the number of parameters. Models with lower BIC scores are preferred to models with higher ones. As pointed out by Raftery (1995), differences in BIC scores may be converted into an approximate Bayes factor:

$$
B_{01}^{*}=\exp \left(-\frac{\mathrm{BIC}_{0}-\mathrm{BIC}_{1}}{2}\right),
$$

where $\mathrm{BIC}_{0}$ and $\mathrm{BIC}_{1}$ are the $\mathrm{BIC}$ scores for the null and the alternative, respectively. For the one-sample case, it is straightforward to show that

$$
\mathrm{BIC}_{0}-\mathrm{BIC}_{1}=N \log \left(1+\frac{t^{2}}{N-1}\right)-\log N
$$

Consequently,

$$
B_{01}^{*}=\sqrt{N}\left(1+\frac{t^{2}}{N-1}\right)^{-N / 2} .
$$

We have included critical $t$ values for $B^{*}=1 / 10(10: 1$ favoring the alternative) in Figure 4. As can be seen, the BIC behaves well for large samples.

$\mathrm{BIC}$ is an asymptotic approximation to a Bayes factor with certain priors (Raftery, 1995). For the one-sample case, these priors are

$$
\begin{aligned}
\sigma^{2} & \sim \operatorname{Normal}\left[\hat{\sigma}^{2},\left(\hat{\sigma}^{2}\right)^{2} / 2\right], \\
\mu & \sim \operatorname{Normal}\left(\hat{\mu}, \hat{\sigma}^{2}\right),
\end{aligned}
$$

where $\hat{\mu}=\bar{y}$ and $\hat{\sigma}^{2}=(1 / N) \Sigma\left(y_{i}-\bar{y}\right)^{2}$ are the maximum likelihood estimators of $\mu$ and $\sigma^{2}$, respectively. These priors are more informative than the ones we advocate. There are two main differences: (1) The prior variance on $\mu$ is the sample variance. In Bayesian inference, it is not valid to specify a prior that depends on the observed data. The justification for this prior is that it is a convenient large-sample approximation for a unit-information prior. Hence, it may only be interpreted for large-sized samples. The JZS and unit-information priors, on the other hand, do not depend on the observed data. As a consequence, the resulting Bayes factors may be interpreted with confidence for all sample sizes. (2) Because BIC approximates a unit-information prior, it is slightly more informative than the JZS prior. As such, the resulting BIC values will slightly favor the alternative more than the JZS Bayes factor would.

BIC is not well-suited for mixed models, such as within-subjects ANOVA. The problem is that BIC is based on counting parameters. The more parameters a model has, the more it is penalized for complexity. In standard between-subjects designs with fixed effects, the number of parameters is unambiguous. In mixed designs, however, each participant is modeled as a random effect that is neither entirely free (the effects must conform to a particular distribution) nor heavily constrained. It is not obvious how these random effects are to be counted or penalized in BIC. Bayes factors also penalize complex models, but they do so without recourse to counting parameters. Instead, complex models are penalized by the diversity of data patterns they explain (Myung \& Pitt, 1997). It is known that JZS priors extend well to simple random-effects models (García-Donato \& Sun, 2007), and we anticipate that they may be used more generally in mixed models, especially with within-subjects factorial designs.

\section{General Discussion}

There has been a long-lasting and voluminous debate in both psychology and statistics on the value of significance tests. Most of this debate has centered on the proper way to test for effects. We advocate a different focus: Psychologists should search for theoretically interesting invariances or regularities in data. Conventional significance tests are ill-suited for stating evidence for 
invariances and, as a consequence, overstate the evidence against them.

It is reasonable to ask whether hypothesis testing is always necessary. In many ways, hypothesis testing has been employed in experimental psychology too often and too hastily, without sufficient attention to what may be learned by exploratory examination for structure in data (Tukey, 1977). To observe structure, it is often sufficient to plot estimates of appropriate quantities along with measures of estimation error (Rouder \& Morey, 2005). As a rule of thumb, hypothesis testing should be reserved for those cases in which the researcher will entertain the null as theoretically interesting and plausible, at least approximately.

Researchers willing to perform hypothesis testing must realize that the endeavor is inherently subjective (Berger \& Berry, 1988). For any data set, the null will be superior to some alternatives and inferior to others. Therefore, it is necessary to commit to specific alternatives, with the resulting evidence dependent to some degree on this commitment. This commitment is essential to and unavoidable for sound hypothesis testing in both frequentist and Bayesian settings. We advocate Bayes factors because their interpretation is straightforward and natural. Moreover, in Bayesian analysis, the elements of subjectivity are transparent rather than hidden (Wagenmakers, Lee, Lodewyckx, \& Iverson, 2008).

This commitment to specify judicious and reasoned alternatives places a burden on the analyst. We have provided default settings appropriate to generic situations. Nonetheless, these recommendations are just that and should not be used blindly. Moreover, analysts can and should consider their goals and expectations when specifying priors. Simply put, principled inference is a thoughtful process that cannot be performed by rigid adherence to defaults.

There is no loss in dispensing with the illusion of objectivity in hypothesis testing. Researchers are acclimated to elements of social negotiation and subjectivity in scientific endeavors. Negotiating the appropriateness of various alternatives is no more troubling than negotiating the appropriateness of other elements, including design, operationalization, and interpretation. As part of the everyday practice of psychological science, we have the communal infrastructure to evaluate and critique the specification of alternatives. This view of negotiated alternatives is vastly preferable to the current practice, in which significance tests are mistakenly regarded as objective. Even though inference is subjective, we can agree on the boundaries of reasonable alternatives. The sooner we adopt inference based on specifying alternatives, the better.

\section{AUTHOR NOTE}

We are grateful for valuable comments from Michael Pratte, E.-J. Wagenmakers, and Peter Dixon. This research was supported by NSF Grant SES-0720229 and NIMH Grant R01-MH071418. Please address correspondence to J. N. Rouder, Department of Psychological Sciences, 210 McAlester Hall, University of Missouri, Columbia, MO 65211 (e-mail: rouderj@missouri.edu).

\section{REFERENCES}

AKaIKe, H. (1974). A new look at the statistical model identification. IEEE Transactions on Automatic Control, 19, 716-723.
Ashby, F. G., \& Maddox, W. T. (1992). Complex decision rules in categorization: Contrasting novice and experienced performance. Journal of Experimental Psychology: Human Perception \& Performance, 18, 50-71.

Augustin, T. (2008). Stevens' power law and the problem of meaningfulness. Acta Psychologica, 128, 176.

Berger, J. O., \& Berry, D. A. (1988). Analyzing data: Is objectivity possible? American Scientist, 76, 159-165.

Bishop, Y. M. M., FienberG, S. E., \& Holland, P. W. (1975). Discrete multivariate analysis: Theory and practice. Cambridge, MA: MIT Press.

Clarke, F. R. (1957). Constant-ratio rule for confusion matrices in speech communication. Journal of the Acoustical Society of America, 29, 715-720.

Cohen, J. (1994). The earth is round $(p<.05)$. American Psychologist, 49, 997-1003.

Cumming, G., \& Finch, S. (2001). A primer on the understanding, use, and calculation of confidence intervals based on central and noncentral distributions. Educational \& Psychological Measurement, 61, 532-574.

Debner, J. A., \& JACOBY, L. L. (1994). Unconscious perception: Attention, awareness, and control. Journal of Experimental Psychology: Learning, Memory, \& Cognition, 20, 304-317.

Dehaene, S., Naccache, L., Le Clec'H, G., Koechlin, E., MuelLer, M., Dehaene-Lambertz, G., ET al. (1998). Imaging unconscious semantic priming. Nature, 395, 597-600.

Edwards, W., Lindman, H., \& Savage, L. J. (1963). Bayesian statistical inference for psychological research. Psychological Review, 70, 193-242.

Egan, J. P. (1975). Signal detection theory and ROC-analysis. New York: Academic Press.

FeCHNER, G. T. (1966). Elements of psychophysics. New York: Holt, Rinehart \& Winston. (Original work published 1860)

García-Donato, G., \& Sun, D. (2007). Objective priors for hypothesis testing in one-way random effects models. Canadian Journal of Statistics, 35, 303-320.

Gelman, A., Carlin, J. B., Stern, H. S., \& Rubin, D. B. (2004). Bayesian data analysis (2nd ed.). Boca Raton, FL: Chapman \& Hall.

Gillispie, C. C., Fox, R., \& Grattan-Guinness, I. (1997). PierreSimon Laplace, 1749-1827: A life in exact science. Princeton, NJ: Princeton University Press.

Gönen, M., Johnson, W. O., Lu, Y., \& Westfall, P. H. (2005). The Bayesian two-sample $t$ test. American Statistician, 59, 252-257.

Goodman, S. N. (1999). Toward evidence-based medical statistics: I. The $p$ value fallacy. Annals of Internal Medicine, 130, 995-1004.

Green, D. M., \& Swets, J. A. (1966). Signal detection theory and psychophysics. New York: Wiley.

Grider, R. C., \& Malmberg, K. J. (2008). Discriminating between changes in bias and changes in accuracy for recognition memory of emotional stimuli. Memory \& Cognition, 36, 933-946.

HAWKING, S. (ED.) (2002). On the shoulders of giants: The great works of physics and astronomy. Philadelphia: Running Press.

HaYs, W. L. (1994). Statistics (5th ed.). Fort Worth, TX: Harcourt Brace.

JACOBY, L. L. (1991). A process dissociation framework: Separating automatic from intentional uses of memory. Journal of Memory \& Language, 30, 513-541.

JefFreys, H. (1961). Theory of probability (3rd ed.). Oxford: Oxford University Press, Clarendon Press.

Kass, R. E., \& RAfTery, A. E. (1995). Bayes factors. Journal of the American Statistical Association, 90, 773-795.

Kass, R. E., \& Wasserman, L. (1995). A reference Bayesian test for nested hypotheses with large samples. Journal of the American Statistical Association, 90, 928-934.

Killeen, P. R. (2005). An alternative to null-hypothesis significance tests. Psychological Science, 16, 345-353.

Killeen, P. R. (2006). Beyond statistical inference: A decision theory for science. Psychonomic Bulletin \& Review, 13, 549-562.

Kline, R. B. (2004). Beyond significance testing: Reforming data analysis methods in behavioral research. Washington, DC: American Psychological Association.

LeE, M. D., \& WagenMakers, E.-J. (2005). Bayesian statistical inference in psychology: Comment on Trafimow (2003). Psychological Review, 112, 662-668. 
Lehmann, E. L. (1993). The Fisher, Neyman-Pearson theories of testing hypotheses: One theory or two? Journal of the American Statistical Association, 88, 1242-1249.

Liang, F., Paulo, R., Molina, G., Clyde, M. A., \& Berger, J. O. (2008). Mixtures of $g$ priors for Bayesian variable selection. Journal of the American Statistical Association, 103, 410-423.

Lindley, D. V. (1957). A statistical paradox. Biometrika, 44, 187-192.

Logan, G. D. (1988). Toward an instance theory of automatization. Psychological Review, 95, 492-527.

LogAN, G. D. (1992). Shapes of reaction-time distributions and shapes of learning curves: A test of the instance theory of automaticity. Journal of Experimental Psychology: Learning, Memory, \& Cognition, 18, 883-914.

LuCE, R. D. (1959). Individual choice behavior: A theoretical analysis. New York: Wiley.

Masson, M. E. J., \& LofTus, G. R. (2003). Using confidence intervals for graphically based data interpretation. Canadian Journal of Experimental Psychology, 57, 203-220.

MeEhl, P. E. (1978). Theoretical risks and tabular asterisks: Sir Karl, Sir Ronald, and the slow progress of soft psychology. Journal of Consulting \& Clinical Psychology, 46, 806-834.

MyUnG, I.-J., \& PiTt, M. A. (1997). Applying Occam's razor in modeling cognition: A Bayesian approach. Psychonomic Bulletin \& Review, 4, 79-95.

Plant, E. A., \& Peruche, B. M. (2005). The consequences of race for police officers' responses to criminal suspects. Psychological Science, 16, 180-183.

RAFTERY, A. E. (1995). Bayesian model selection in social research. Sociological Methodology, 25, 111-163.

Reingold, E. M., \& Merikle, P. M. (1988). Using direct and indirect measures to study perception without awareness. Perception \& Psychophysics, 44, 563-575.

Rouder, J. N., \& LU, J. (2005). An introduction to Bayesian hierarchical models with an application in the theory of signal detection. Psychonomic Bulletin \& Review, 12, 573-604.

Rouder, J. N., \& Morey, R. D. (2005). Relational and arelational confidence intervals: A comment on Fidler, Thomason, Cumming, Finch, and Leeman (2004). Psychological Science, 16, 77-79.
Rouder, J. N., Morey, R. D., Speckman, P. L., \& Pratte, M. S (2007). Detecting chance: A solution to the null sensitivity problem in subliminal priming. Psychonomic Bulletin \& Review, 14, 597 605.

Rouder, J. N., \& RatClifF, R. (2004). Comparing categorization models. Journal of Experimental Psychology: General, 133, 63-82.

Schwarz, G. (1978). Estimating the dimension of a model. Annals of Statistics, 6, 461-464.

Sellke, T., Bayarri, M. J., \& Berger, J. O. (2001). Calibration of $p$ values for testing precise null hypotheses. American Statistician, 55, 62-71.

SHEPARD, R. N. (1957). Stimulus and response generalization: A stochastic model relating generalization to distance in psychological space. Psychometrika, 22, 325-345.

Shibley Hyde, J. (2005). The gender similarities hypothesis. American Psychologist, 60, 581-592.

Shibley Hyde, J. (2007). New directions in the study of gender similarities and differences. Current Directions in Psychological Science, 16, 259-263.

Stevens, S. S. (1957). On the psychophysical law. Psychological Review, 64, 153-181.

Swets, J. A. (1996). Signal detection theory and ROC analysis in psychology and diagnostics: Collected papers. Mahwah, NJ: Erlbaum.

TuKEY, J. W. (1977). Exploratory data analysis. Reading, MA: AddisonWesley.

Wagenmakers, E.-J. (2007). A practical solution to the pervasive problem of $p$ values. Psychonomic Bulletin \& Review, 14, 779-804.

WAGENMAKERS, E.-J., \& GRÜNWALD, P. (2006). A Bayesian perspective on hypothesis testing: A comment on Killeen (2005). Psychological Science, 17, 641-642.

Wagenmakers, E.-J., Lee, M. D., Lodewyckx, T., \& Iverson, G. (2008). Bayesian versus frequentist inference. In H. Hoijtink, I. Klugkist, \& P. A. Boelen (Eds.), Bayesian evaluation of informative hypotheses in psychology (pp. 181-207). New York: Springer.

Zellner, A., \& Siow, A. (1980). Posterior odds ratios for selected regression hypotheses. In J. M. Bernardo, M. H. DeGroot, D. V. Lindley, \& A. F. M. Smith (Eds.), Bayesian statistics: Proceedings of the First International Meeting (pp. 585-603). Valencia: University of Valencia Press.

\section{NOTES}

1. The calculation of the standard deviation of $y_{i}$ assumes that there are no participant-by-item interactions. This assumption is made for computational convenience, and the presence of such interactions does not threaten the validity of the argument that significance tests overstate the evidence against the null hypothesis.

2 . For the case in which the alternative is a point and $\sigma$ is known,

$$
M_{0}=\left(2 \pi \sigma^{2}\right)^{-N / 2} \exp \left(-\frac{\sum y_{i}^{2}}{2 \sigma^{2}}\right), M_{1}=\left(2 \pi \sigma^{2}\right)^{-N / 2} \exp \left(-\frac{\sum\left(y_{i}-\mu_{1}\right)^{2}}{2 \sigma^{2}}\right) .
$$

The Bayes factor is given by

$$
B_{01}=\frac{M_{0}}{M_{1}}=\exp \left[-\frac{N \mu_{1}}{2 \sigma^{2}}\left(2 \bar{y}-\mu_{1}\right)\right] .
$$

This Bayes factor is a function of $\bar{y} / \sigma$, the observed effect size, and $\mu_{1} / \sigma$, the effect size of the alternative.

3. The Bayes factor with the normal prior is

$$
B_{01}=\left(\phi \frac{\sigma_{\mu}^{2}}{\sigma^{2}}\right)^{1 / 2} \exp \left(-\frac{N^{2} \bar{y}^{2}}{2 \phi \sigma^{2}}\right)
$$

where

$$
\phi=N+\frac{\sigma^{2}}{\sigma_{\mu}^{2}} .
$$

This Bayes factor depends only on the observed effect size $\bar{y} / \sigma$ and the ratio $\sigma_{\mu} / \sigma$. 
4. The scaled JZS Bayes factor is

$$
B_{01}=\frac{\left(1+\frac{t^{2}}{v}\right)^{-(v+1) / 2}}{\int_{0}^{\infty}\left(1+N g r^{2}\right)^{-1 / 2}\left(1+\frac{t^{2}}{\left(1+N g r^{2}\right) v}\right)^{-(v+1) / 2}(2 \pi)^{-1 / 2} g^{-3 / 2} e^{-1 /(2 g)} d g},
$$

where $r$ is the scale factor.

5. The scaled-information Bayes factor is

$$
B_{01}=\frac{\left(1+\frac{t^{2}}{v}\right)^{-(v+1) / 2}}{\left(1+N r^{2}\right)^{-1 / 2}\left(1+\frac{t^{2}}{\left(1+N r^{2}\right) v}\right)^{-(v+1) / 2}}
$$

where $r$ is the scale factor. The unit-information Bayes factor holds when $r=1$.

6. There is a more principled Bayes factor calculation for those who believe that the null can never be true a priori. The null may be specified as a composite - that is, as a distribution of effect sizes. A reasonable choice is that under the null, the effect size is normally distributed with a mean of 0 and a small standard deviation of, say, .05. If this standard deviation is much smaller than that for the alternative, then the JZS Bayes factor serves as a suitable approximation for moderate sample sizes.

(Manuscript received June 4, 2008;

revision accepted for publication August 27, 2008.) 